\title{
Cerebrospinal Fluid Clearance in Alzheimer Disease Measured with Dynamic PET
}

\author{
Mony J. de Leon*1, Yi Li*1, Nobuyuki Okamura ${ }^{2}$, Wai H. Tsui ${ }^{1}$, Les A. Saint-Louis ${ }^{3}$, Lidia Glodzik ${ }^{1,4}$, Ricardo S. Osorio ${ }^{1}$, \\ Juan Fortea $^{5}$, Tracy Butler ${ }^{1}$, Elizabeth Pirraglia ${ }^{1}$, Silvia Fossati ${ }^{1,6}$, Hee-Jin Kim ${ }^{1,7}$, Roxana O. Carare ${ }^{8}$, \\ Maiken Nedergaard ${ }^{9,10}$, Helene Benveniste ${ }^{11}$, and Henry Rusinek*4 \\ ${ }^{I}$ Department of Psychiatry, New York University School of Medicine, Center for Brain Health, New York, New York; ${ }^{2}$ Department of \\ Pharmacology, Tohoku University School of Medicine, Tohoku, Japan; ${ }^{3}$ Manhattan Diagnostic Radiology, New York, New York; ${ }^{4}$ Department \\ of Radiology, New York University Center School of Medicine, New York, New York; ${ }^{5}$ Memory Unit, Department of Neurology, Hospital de la \\ Santa Creu i Sant Pau, Biomedical Research Institute Sant Pau, Universitat Autònoma de Barcelona, Barcelona, Spain; ${ }^{6}$ Department of \\ Neurology, New York University School of Medicine, New York, New York; ${ }^{7}$ Department of Neurology, College of Medicine, Hanyang \\ University, Seoul, Korea; ${ }^{8}$ Faculty of Medicine, University of Southampton, Southampton, United Kingdom; ${ }^{9}$ Center for Translational \\ Neuromedicine, University of Rochester Medical Center, Rochester, New York; ${ }^{10}$ Center for Basic and Translational Neuroscience, University \\ of Copenhagen, Copenhagen, Denmark; and ${ }^{11}$ Department of Anesthesiology, Yale School of Medicine, New Haven, Connecticut
}

Evidence supporting the hypothesis that reduced cerebrospinal fluid (CSF) clearance is involved in the pathophysiology of Alzheimer disease $(A D)$ comes primarily from rodent models. However, unlike rodents, in which predominant extracranial CSF egress is via olfactory nerves traversing the cribriform plate, human CSF clearance pathways are not well characterized. Dynamic PET with ${ }^{18} \mathrm{~F}-\mathrm{THK} 5117$, a tracer for tau pathology, was used to estimate the ventricular CSF time-activity as a biomarker for CSF clearance. We tested 3 hypotheses: extracranial CSF is detected at the superior turbinates; CSF clearance is reduced in AD; and CSF clearance is inversely associated with amyloid deposition. Methods: Fifteen subjects, 8 with $A D$ and 7 normal control volunteers, were examined with ${ }^{18} \mathrm{~F}-\mathrm{THK} 5117$. Ten subjects additionally underwent ${ }^{11} \mathrm{C}$-Pittsburgh compound $\mathrm{B}\left({ }^{11} \mathrm{C}\right.$-PiB) PET scanning, and 8 were ${ }^{11} \mathrm{C}$-PiB-positive. Ventricular time-activity curves of ${ }^{18} \mathrm{~F}-\mathrm{THK} 5117$ were used to identify highly correlated time-activity curves from extracranial voxels. Results: For all subjects, the greatest density of CSF-positive extracranial voxels was in the nasal turbinates. Tracer concentration analyses validated the superior nasal turbinate CSF signal intensity. AD patients showed ventricular tracer clearance reduced by $23 \%$ and $66 \%$ fewer superior turbinate CSF egress sites. Ventricular CSF clearance was inversely associated with amyloid deposition. Conclusion: The human nasal turbinate is part of the CSF clearance system. Lateral ventricle and superior nasal turbinate CSF clearance abnormalities are found in AD. Ventricular CSF clearance reductions are associated with increased brain amyloid depositions. These data suggest that PET-measured CSF clearance is a biomarker of potential interest in $A D$ and other neurodegenerative diseases.

Key Words: neurology; PET/CT; research methods; Alzheimer disease; CSF clearance; dynamic PET; THK5117

J Nucl Med 2017; 58:1471-1476

DOI: 10.2967/jnumed.116.187211

Received Nov. 14, 2016; revision accepted Feb. 27, 2017.

For correspondence contact: Mony de Leon, New York University, Department of Psychiatry, Center for Brain Health, 145 East 32 St., New York, NY 10016.

E-mail: mony.deleon@nyumc.org

${ }^{*}$ Contributed equally to this work.

Published online Mar. 16, 2017.

COPYRIGHT (C) 2017 by the Society of Nuclear Medicine and Molecular Imaging.
$\mathbf{I}_{\mathrm{n}}$ mpairments in cerebrospinal fluid (CSF) turnover have been suspected for many years in the etiology of Alzheimer disease (AD) and propagation of amyloid- $\beta$ (A $\beta$ ) lesions (1-4). However, there are no noninvasive imaging tools to evaluate CSF clearance.

Impaired CSF clearance is observed in mouse aging (5) and AD (6) models. In rodents and other mammals, CSF is primarily cleared along olfactory nerves that traverse the cribriform plate, draining into lymphatic vessels in the nasal mucosa (7-10). Rodent CSF clearance is rapid because radiolabeled albumin (11), Indian ink (7), and paramagnetic contrast (10) injected into the CSF reach the turbinates within minutes. Much less is known about human CSF clearance anatomy. Arachnoid granulations, arguably considered important human CSF egress sites (12), are not found in the rodent. The only evidence for a human nasal turbinate CSF efflux pathway comes from postmortem studies $(13,14)$.

Low-molecular-weight radiotracers enter and egress the CSF through the choroid plexus and perivascular drainage (12), yet CSF time-activity curves have not been explored in the PET literature. Using in vivo dynamic PET with ${ }^{18} \mathrm{~F}-\mathrm{THK} 5117$ and ${ }^{11} \mathrm{C}$-Pittsburgh compound $\mathrm{B}\left({ }^{11} \mathrm{C}-\mathrm{PiB}\right)$ tracers in healthy elderly and $\mathrm{AD}$ subjects, we estimated ventricular CSF time-activity curves to confirm 3 hypotheses: CSF is cleared via the superior nasal turbinates; CSF clearance is reduced in AD; and the magnitude of $\mathrm{A} \beta$ binding is inversely associated with the CSF clearance.

\section{MATERIALS AND METHODS}

\section{Participants and Clinical Assessments}

With ethics committee approval, written informed consent was obtained from 15 participants or their legal care takers. Eight probable $\mathrm{AD}$ patients were recruited from the memory clinic of Tohoku University Hospital, and 7 healthy normal (NL) volunteer controls were recruited from the community (Table 1). All subjects were diagnosed at a consensus conference, and the diagnosis of $\mathrm{AD}$ was made in accordance with the criteria of the National Institute of Neurologic and Communicative Disorders and Stroke/Alzheimer Disease and Related Disorders Association (15). All participants underwent high-resolution T1-weighted MRI and PET examination with the ${ }^{18} \mathrm{~F}$-THK5117 tracer. Ten of these subjects, $5 \mathrm{NL}$ and $5 \mathrm{AD}$, also underwent ${ }^{11} \mathrm{C}$-PiB PET scanning. 
TABLE 1

Study Subjects

\begin{tabular}{lcc}
\hline \multicolumn{1}{c}{ Characteristic } & NL $(n=7)$ & AD $(n=8)$ \\
\hline Age $(y)$ & $74.1 \pm 5.6$ (range, $67-83)$ & $79.8 \pm 10.6($ range, $57-89)$ \\
\hline Education $(y)$ & $14.6 \pm 2.2$ & $12.1 \pm 2.7$ \\
Male/female $(n)$ & $4 / 3$ & $2 / 6$ \\
MMSE & $28.7 \pm 1.6$ & $18.1 \pm 4.6^{*}$ \\
CDR & $0.0 \pm 0.0$ & $2.0 \pm 0.8^{*}$ \\
Disease duration $(y)$ & - & $5.3 \pm 1.7$ \\
& & \\
\hline MMSE $=$ Mini-Mental State Examination; CDR $=$ Clinical Dementia Rating. & \\
Values are mean \pm SD, ${ }^{*} P<0.05$. &
\end{tabular}

\section{Image Acquisition and Processing}

Dynamic PET Acquisition. The PET radiotracers were administered in 2 imaging sessions within 2 wk. The radiotracers included ${ }^{18} \mathrm{~F}$ THK5117, a 2-arylquinoline derivative validated for neurofibrillary pathology (16), and ${ }^{11} \mathrm{C}-\mathrm{PiB}$, an analog of thioflavin- $\mathrm{T}$ validated for brain $\mathrm{A} \beta$ plaques (17). Both tracers were prepared at the Cyclotron and Radioisotope Center of Tohoku University, both have low molecular weight $(<328 \mathrm{~g} / \mathrm{mole})$, and both freely cross the blood-brain barrier $(16,18) .{ }^{18} \mathrm{~F}-\mathrm{THK} 5117,185 \mathrm{mBq}$, and ${ }^{11} \mathrm{C}-\mathrm{PiB}, 296 \mathrm{mBq}$, were administered by intravenous bolus injection. Dynamic PET data were acquired axially along the canthomeatal plane in list mode continuously up to 90 min using a SET-3000 G/X PET scanner (Shimadzu (19)). The supplemental materials (available at http://jnm.snmjournals.org) list the timing of the reconstructed PET image frames. The scanner is equipped with high-energy resolution germanium oxyorthosilicate scintillators that provide $4.5-\mathrm{mm}$ (transverse) and 5.4-mm (axial) spatial resolutions (full width at half maximum, at $10 \mathrm{~cm}$ off axis). Attenuation correction was based on a transmission image from a rotating ${ }^{137} \mathrm{Cs}$ point source. The field of view superiorly encompassed the skull and inferiorly the foramen magnum and maxilla.

MRI Acquisition. Each subject underwent MRI on a SIGNA $1.5 \mathrm{~T}$ magnet (GE Healthcare). The protocol included a T1-weighted spoiled gradient recalled echo axial sequence with parameters echo time/repetition time, 2.4/22 ms; flip angle, $20^{\circ}$; acquisition matrix, $256 \times 256 \times 110$; 1 excitation; axial field of view, $22 \mathrm{~cm}$; bandwidth, $122 \mathrm{~Hz} / \mathrm{px}$; voxel size, $0.86 \times 0.86 \times$ 1.0. In all cases, the MRI field of view covered the PET field of view.

PET Image Workflow. PET data were reconstructed to a $128 \times 128 \times$ 79 matrix of $2 \times 2 \times 2.6 \mathrm{~mm}$ voxels. After decay correction and SUV normalization, each dynamic PET frame was coregistered with the corresponding T1-weighted MR image using statistical parametric mapping (SPM 12) software (www.fil.ion.ucl.ac.uk/spm). PET images

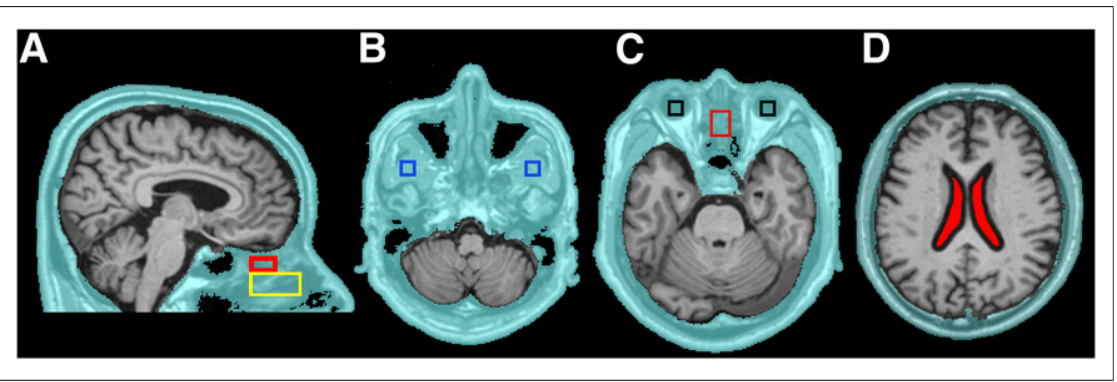

FIGURE 1. Extracranial shell and ROIs. Extracranial shell region (A-D) is in aqua. The following subregions were examined: superior (red) and middle turbinates (yellow) (A), pterygoid muscle (blue) (B), globes of the eye (black) and superior turbinate (red) (C), and ventricular CSF (filled red) (D). were sampled in their original space and partial volume corrected using a modified 1-tissue model (20).

MRI Regional Segmentation. To explore CSF egress, an extracranial shell region of interest (ROI) was defined on MRI. The shell included scalp, bone, and soft tissues (muscle, nasal turbinates, and globes of the eye) and extended inferiorly to the foramen magnum and maxilla. It excluded brain, subarachnoid CSF, and air (Fig. 1). The following shell ROIs were drawn on the MRI by a reader masked to the PET data. (1) The superior nasal turbinates were sampled at a distance $5.2 \mathrm{~mm}$ ( 2 voxels) inferior to the frontal lobe to minimize partial-volume effects from the brain. The anterior boundary was at the crista galli. The ROI dimensions were $18 \mathrm{~mm}$ anterior-posterior (A-P), $14 \mathrm{~mm}$ left-right (L-R), and $10 \mathrm{~mm}$ craniocaudal. (2) The middle nasal turbinates were sampled inferior to the superior turbinate with the following ROI dimensions: $34 \mathrm{~mm}$ A-P, $16 \mathrm{~mm} \mathrm{L-R}$, and $18 \mathrm{~mm}$ craniocaudal. (3) The globes of the eyes and lateral pterygoid muscle were sampled bilaterally and both ROIs were $12 \mathrm{~mm} \mathrm{A-P,} 12 \mathrm{~mm} \mathrm{L-R,} \mathrm{and} 6 \mathrm{~mm}$ cranio-caudal.

The MRI brain was segmented into lateral ventricle, neocortical gray and white matter, and cerebellar hemisphere gray matter using Free-Surfer (version 5.1, http://surfer.nmr.mgh.harvard.edu). The ventricular CSF ROI was shrunk by 2 voxels $(5.2 \mathrm{~mm})$ to minimize partial-volume contamination from brain tissue (Fig. 1D). Imagederived arterial blood tracer concentrations were sampled from the internal carotid artery (21) and venous blood sampled at the junction of the superior sagittal and transverse sinus.

Mapping CSF Clearance in Shell. CSF-positive voxels were identified in each subject using a seeding procedure similar to one used to identify neural networks in resting functional MRI (22). The time-activity curve of every shell voxel was correlated (Pearson Product $r$ ) with the time-activity curve from the ventricular CSF (supplemental materials). Voxels with correlations of $r>0.95$ were considered potentially CSF-positive and further tested for tracer concentrations.

Tracer Concentration Estimations and CSF Validation. Voxel tracer concentrations were derived from the SUV data normalized by the corresponding cerebellar gray matter time frames and were examined as area under the curve (AUC) and as the rate of change. For THK5117, the 35- to 80-min time period $\left(\mathrm{AUC}_{35-80} \mathrm{~min}\right)$ was selected because blood concentrations were consistently low and confirmed in another study (23). The CSF validation for each shell region was determined by contrasting the average tracer concentrations of the positive shell voxels, 


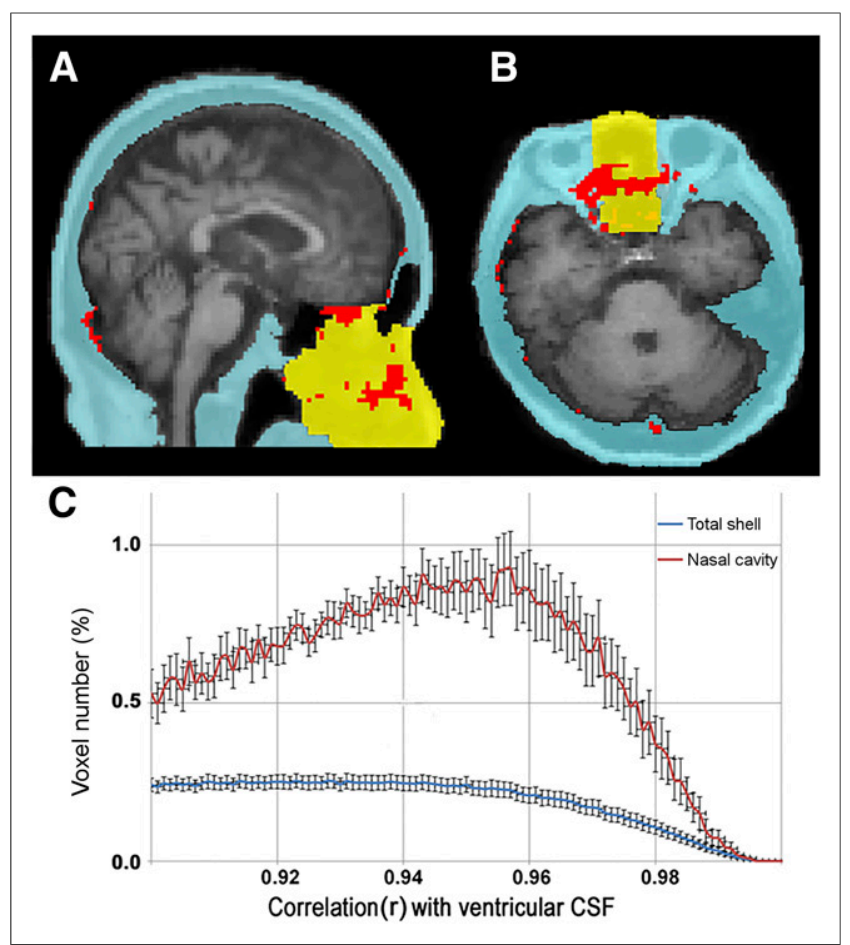

FIGURE 2. Extracranial distribution of CSF time-activity curve correlated voxels. Sagittal (A) and axial (B) MR images from a representative NL show 3-dimensional extracranial shell region in blue and yellow (total nasal cavity). (C) Percentage distribution ( \pm SEM) $(n=5$ subjects) of shell and nasal cavity voxels whose tau PET derived time-activity curve are correlated with ventricular CSF time-activity curve, within range of $r=$ 0.90-0.99. Data show 3-fold-greater percentage of CSF-correlated voxels in nasal cavity than in total shell. Shell voxels correlated at $r>0.95$ were considered CSF-positive and are mapped in red in A and B.

against tracer concentrations from ventricular CSF, blood, and brain.

Measures of Tracer Binding. Conventional time frames were used to estimate tracer binding, 50-80 $\mathrm{min}$ for ${ }^{18} \mathrm{~F}-\mathrm{THK} 5117$ and 50 $70 \mathrm{~min}$ for ${ }^{11} \mathrm{C}-\mathrm{PiB}$. Both measures were normalized by their respective cerebellar gray matter SUV to form the SUV ratio (SUVR). SUVR values of 1.5 or more defined positive voxels (24).

\section{Statistical Analysis}

Correlations were examined with Spearman tests and partial correlations using Quade's index of matched correlation (25). Between-group contrasts were made with the Mann-Whitney $U(\mathrm{U})$ test. Within-subject anatomic specificity tests were examined with the related-samples Wilcoxon (W) signed-rank tests. The Holm-Bonferroni method for multiple comparisons was used for $P$ value adjustment (26). All tests were 2-sided, and statistical significance was set at $P<0.05$ when not adjusted for multiple comparisons.

\section{RESULTS}

\section{Study 1: Anatomic Distribution and Validation of Nasal Cavity CSF}

Nasal Cavity CSF. Across all subjects, the time-activity curve correlation analysis showed a 3-fold-greater percentage of CSFpositive extracranial voxels (red) in the nasal cavity (yellow) than in the rest of the total shell in blue (Fig. 2).

ROI Analyses. All subjects had CSF-positive voxels in the superior turbinate ROI (Fig. 3). Among the extracranial ROIs, the superior turbinate ROI had the greatest tracer concentration. Both superior and middle turbinate ROIs show greater than $23 \%$ CSFpositive voxel density, whereas the globe of the eye, pterygoid muscle, and total shell showed less than 5\% (Table 2). After partial-volume correction the superior turbinate concentration remained the greater (supplemental materials). A high density of CSF-positive nasal turbinate voxels was confirmed in 4 normal subjects (supplemental materials) using ${ }^{11} \mathrm{C}$-cocaine, a PET tracer with little neocortical uptake, rapid clearance, and no nasal turbinate binding. Supplemental Video 1 depicts for 1 NL the extracranial CSF transit over 9 min.

CSF Validation of Positive Shell Voxels. The CSF origin of the positive superior turbinate voxels was supported by tracer concentration analyses $(n=15)$. The average tracer concentration of the positive turbinate voxels $(1.27 \pm 0.25)$ did not differ from the ventricular CSF $(1.27 \pm 0.34, P>0.05)$. However, positive superior turbinate voxels had significantly lower tracer concentrations than brain $(2.69 \pm 0.40, \mathrm{~W}=3.41, P<0.01)$ and were higher than blood (arterial: $0.94 \pm 0.31, \mathrm{~W}=-2.56, P \leq 0.01$, and venous: $0.96 \pm 0.22, \mathrm{~W}=-2.33, P<0.05$; Fig. 4 ).

\section{Study 2: CSF Clearance in $A D$}

The ventricular $\mathrm{AUC}_{35-80}$ min was $23 \%$ lower in $\mathrm{AD}$ than $\mathrm{NL}$ $(0.53 \pm .08$ and $0.69 \pm 0.09$, respectively, $\mathrm{U}=7, P=0.01)$. The rate of ventricular CSF clearance was reduced $33 \%$ in $\mathrm{AD}(0.29 \pm$ 0.08 and $0.43 \pm 0.10$, respectively, $\mathrm{U}=4, P<0.01$; Figs. $5 \mathrm{~A}$ and $5 B)$. Sixty-six percent fewer CSF-positive superior turbinate voxels were found in AD than NL (39.4 \pm 18.5 vs. $115.1 \pm 71.1, \mathrm{U}=$ $7, P=0.01$; Fig. 5C). The significance of these results was unchanged after partial-volume correction.

\section{Study 3: CSF Clearance and Amyloid Positivity}

Eight of 10 subjects, including $5 \mathrm{AD}$ and $3 \mathrm{NL}$, were ${ }^{11} \mathrm{C}-\mathrm{PiB}-$ positive. Both the ventricular $\mathrm{AUC}_{35-80}$ min and the rate of ventricular CSF clearance from THK-5117 were inversely correlated with gray matter amyloid binding $(\rho=0.93, P<0.01$ and $\rho=$ $0.74, P<0.05$, respectively, Fig. 6 ). Demonstrating the precision of the ventricle measurement, the THK5117 $\mathrm{AUC}_{35-80}$ min and ${ }^{11} \mathrm{C}-\mathrm{PiB} \mathrm{AUC}_{35-80}$ min were highly correlated $(\rho=0.83, P<0.01$; supplemental materials). Compared with THK5117, ${ }^{11} \mathrm{C}-\mathrm{PiB}$ showed greater binding in the turbinate cavity (THK-5117

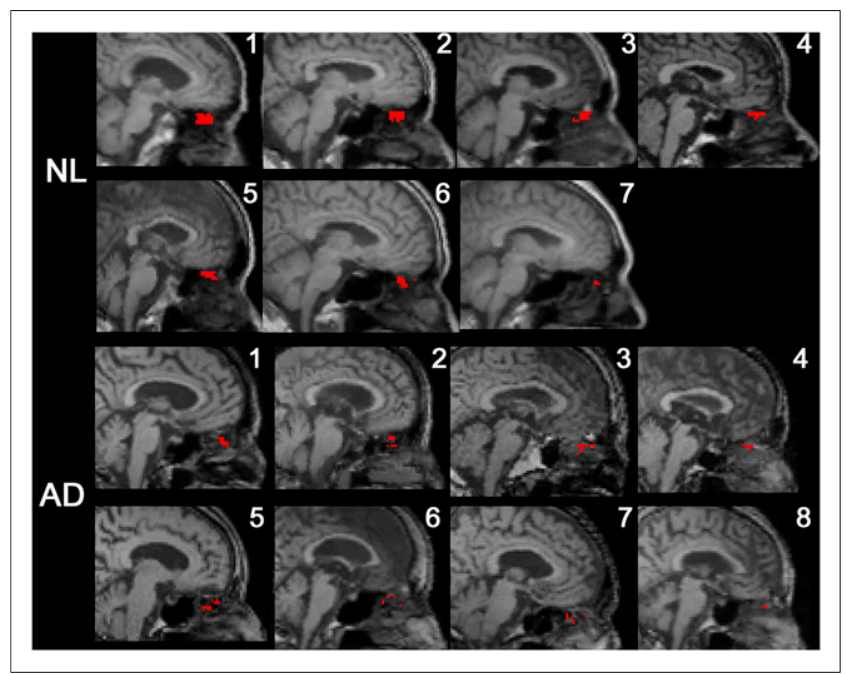

FIGURE 3. Midsagittal MR image with superimposed PET data from all subjects. CSF-positive voxels falling into superior turbinate $\mathrm{ROI}$ are mapped in red. 
TABLE 2

Shell Regions $(n=15)$

\begin{tabular}{|c|c|c|c|c|c|}
\hline Measure & Superior turbinates & Middle turbinates & Eye & Muscle & Shell \\
\hline Number of voxels in ROls & 315 & 1,160 & 360 & 216 & $181,621 \pm 23,086$ \\
\hline Number of positive voxels & $74.7 \pm 61.7$ & $264.9 \pm 194.4$ & $15.4 \pm 13.2$ & $5.62 \pm 5.08$ & $7,139.0 \pm 3,520.8$ \\
\hline ROI positive voxel density (\%) & $23.7 \pm 20.1$ & $22.8 \pm 15.9$ & $4.3 \pm 3.6^{\star}$ & $2.6 \pm 2.3^{\star}$ & $3.9 \pm 2.0^{\star}$ \\
\hline Tracer concentration (SUV $\mathrm{Sean}, 35-80 \mathrm{~min})$ & $1.27 \pm 0.25$ & $1.12 \pm 0.23^{\star}$ & $0.71 \pm 0.58^{\star}$ & $0.86 \pm 0.51^{\star}$ & $1.09 \pm 0.11^{*}$ \\
\hline
\end{tabular}

${ }^{*}$ Different from superior turbinate, Wilcoxon signed-rank test, $P<0.02$.

Values are mean \pm SD.

SUVR $=0.58 \pm 0.12$ and ${ }^{11} \mathrm{C}-\mathrm{PiB}$ SUVR $\left.=1.31 \pm 0.32, n=10\right)$. Therefore, the ${ }^{11} \mathrm{C}-\mathrm{PiB}$ turbinates were not explored.

Examination of Confounds. Five potential confounds were examined. First, elevated tau binding in AD would be expected to reduce the available tau tracer to be cleared to CSF. The extent of this effect was estimated by comparing the ventricular $\mathrm{AUC}_{35-80}$ min after cerebellar normalization, a reference region without specific binding, with whole brain normalization, which includes all specific and nonspecific binding sites (supplemental materials). Relative to cerebellar normalization, whole-brain normalization reduced the AUC by $28 \%$ in normal and $31 \%$ in AD. The between-group AUC differences went from $30 \%$ to $36 \%$ and remained significant ( $\mathrm{U}=5, P<0.01$ ), suggesting a relatively greater between-group tracer clearance effect than tracer binding effect. Similarly, after statistical adjustment for the total brain binding, the between-group ventricular $\mathrm{AUC}_{35-80}$ min effect remained significant $(\mathrm{F}=7.8, P=0.02)$. Second, partial-volume

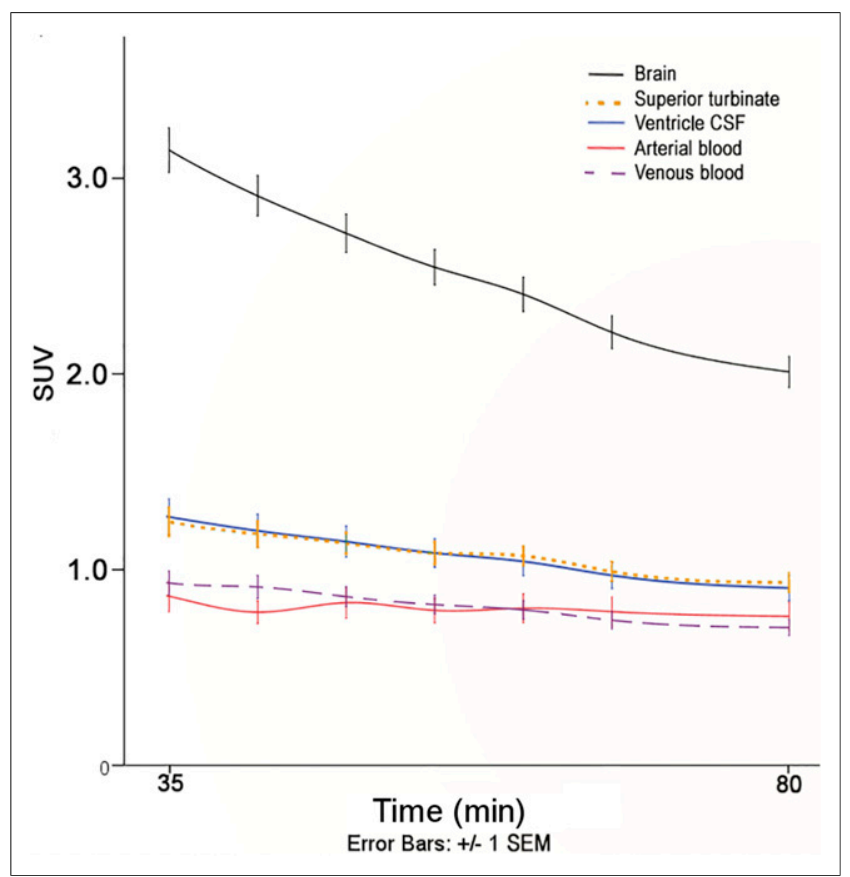

FIGURE 4. Tau tracer concentrations for 5 tissue types over time. Averaged tau tracer counts (SUV) \pm SEM for all study subjects plotted over 35-80 min. Tracer concentrations from CSF-positive superior turbinate and ventricular CSF do not differ from each other, but both are significantly different from blood and brain $(P<0.05)$. errors for the ventricular and extracranial regions were minimized by individually sampling the PET at a 5-mm distance or greater from brain. Additional partial-volume corrections did not change the region or group contrasts. Third, we examined the effect of the larger $\mathrm{AD}$ ventricle $(58.3 \mathrm{~mL} \pm 14.1)$ than normal $(32.2 \mathrm{~mL} \pm$ 11.9, $\mathrm{U}=50, P<0.01)$. After adjustment for ventricular volume, the ventricle $\mathrm{AUC}_{35-80} \mathrm{~min}$ and its rate of change remained lower in $\mathrm{AD}$ (both $\mathrm{F}=5.0, P<0.05$ ). Fourth, no ${ }^{18} \mathrm{~F}-\mathrm{THK} 5117$ binding was observed in the nasal turbinates, and there were no betweengroup turbinate binding differences (SUVR NL $=0.56 \pm 0.10$ and SUVR AD $=0.62 \pm 0.06, P>0.05)$. These data rule out contamination from the nasal mucosa. Fifth, we modeled the effects of reduced flow on the $\mathrm{AUC}_{10-35 \text { min }}$ and $\mathrm{AUC}_{35-80 \mathrm{~min}}$. The results demonstrate negligible flow effects after $10 \mathrm{~min}$, thus supporting our conservative 35-min start point (supplemental materials).

\section{DISCUSSION}

\section{Human Nasal Turbinate CSF Egress}

All nonhuman mammals studied show similar CSF egress pathways that include the choroid plexus, cerebral ventricles, perivascular spaces, and a predominant olfactory nerve drainage site $(12,13,27)$. It is through these pathways that $A \beta$ and other molecules carried in the CSF are removed from the brain $(27,28)$. Human egress sites are poorly understood. It is believed that CSF drains via paraneural pathways and via arachnoid granulations located in the brain and spinal cord (a structure not found in the rodent) (12).

Our results using dynamic PET with ${ }^{18} \mathrm{~F}-\mathrm{THK} 5117$ show that CSF can be detected in the superior nasal turbinates in all subjects. This finding was replicated using ${ }^{11} \mathrm{C}$-cocaine. Our validation study shows that superior turbinate tracer concentrations uniquely differ from other extracranial regions but not from the ventricular CSF concentrations. These results are anatomically consistent with in vivo rodent (11) and postmortem human observations $(13,14)$ demonstrating a CSF egress pathway through the cribriform plate, as well as the rapid intranasal delivery of macromolecules to the CSF (29).

\section{CSF Egress in AD}

Our results show that ventricular CSF clearance is $23 \%$ lower in $\mathrm{AD}$ than $\mathrm{NL}$ and the number of CSF-positive superior turbinate voxels reduced by $66 \%$. Impairments in CSF turnover have been hypothesized for many years in the etiology of $\mathrm{AD}$ and propagation of $A \beta(1-4)$. Unfortunately, neither the animal model nor human evidence is well developed. The pioneering human studies by Bateman et al. (30) used intravenously administered 


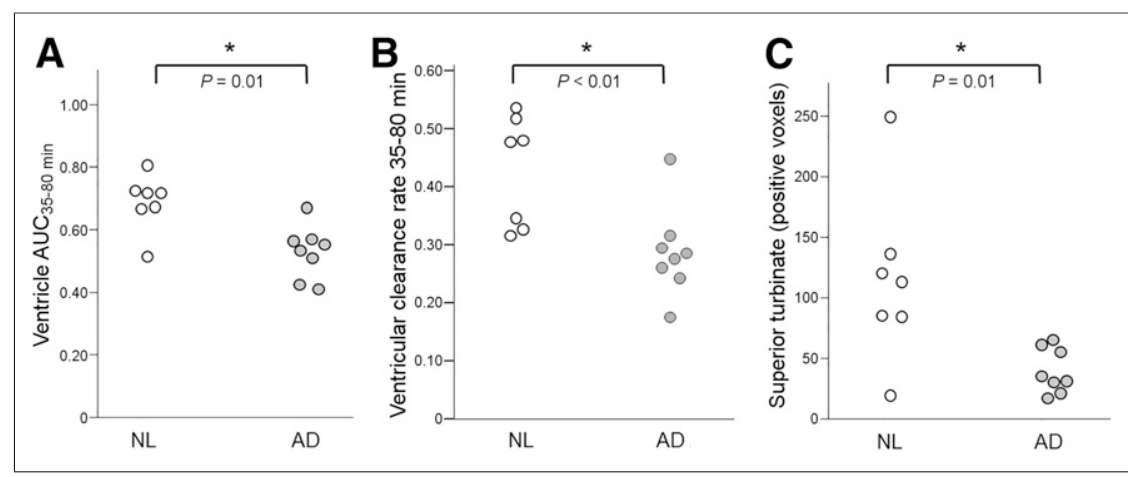

FIGURE 5. Ventricular and superior turbinate CSF clearance in AD. As compared with NL, AD subjects show reduced magnitude of ventricular tracer $\left(A \cup C_{35-80 ~ m i n}\right)(A)$, reduced rate of ventricular tracer clearance (B), and lower number of CSF-positive superior turbinate voxels (C).
Our study has several limitations. The correlated timing of the THK5117 tracer delivery from ventricle to extracranial sites, the basis of our nasal turbinate CSF detection strategy, could be limited by intermittent obstacles to CSF flow. Perivascular $\mathrm{CSF}$ channels in $\mathrm{AD}$ brain accumulate $A \beta(9)$, and specific and nonspecific tracer binding can directly obstruct clearance by binding in clearance sites or by reducing the concentration available for clearance. We selected the $r>0.95$ threshold to maintain precision in identifying extracranial CSF voxels.

Unlike tau tracer AV1451 (38), we found no evidence for THK5117 uptake in choroid plexus. Such uptake would confound the ventricular PET signal. For this

${ }^{13} \mathrm{C}_{6}$-leucine to reveal the time course of labeled $A \beta$ in lumbar puncture-derived CSF. They demonstrated that $\mathrm{A} \beta$ clearance, but not $\mathrm{A} \beta$ production, was reduced in $\mathrm{AD}(31)$. However, their method does not distinguish between impaired membrane transport of $A \beta$ (4) and impaired CSF clearance, which carries the A $\beta$. Suggesting CSF clearance abnormalities in $\mathrm{AD}$, impaired glymphatic CSF clearance was recently observed in aged wild-type mice (5) and in an AD mouse model (6). The known driving forces behind CSF clearance include cardiac output, vasocontractility, and CSF production, facilitated by aquaporin-4 function on egress channels (12). Further, environmental factors such as impaired sleep (32), low activity level (33), and brain inflammation (34) can adversely affect clearance, and all are affected by aging $(12,35,36)$. However, the cause and chronology of impaired CSF clearance and $A \beta$ pathology is unknown. Impaired CSF clearance could both promote and be a consequence of $\mathrm{A} \beta$ pathology. Furthermore, surgical CSF drainage therapy failed to show cognitive benefit in an $\mathrm{AD}$ population, highlighting the complexity of these relationships (37). Nevertheless, our cross-sectional observation of reduced CSF clearance in $\mathrm{AD}$ and of an inverse association between reduced CSF clearance and elevated brain $\mathrm{A} \beta$ levels presents the first, to our knowledge, in vivo support for the hypothesis. Longitudinal study is needed to validate the hypothesis and sort out the chronologic relationships between reduced $\mathrm{CSF}$ clearance and brain $\mathrm{A} \beta$ plaques.

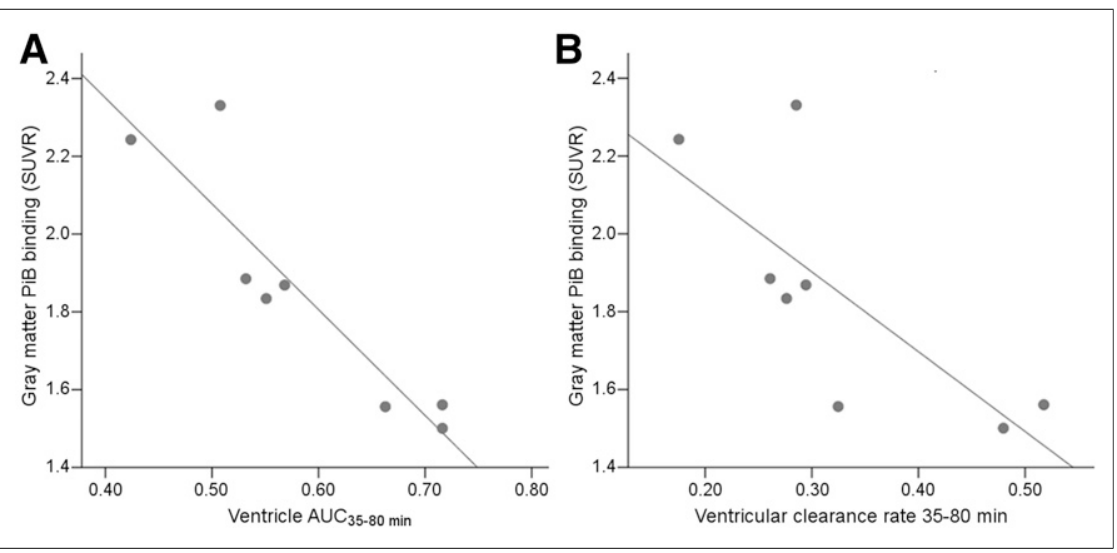

FIGURE 6. Amyloid binding and CSF clearance: relationship, for $8{ }^{11} \mathrm{C}-\mathrm{PiB}-$ positive subjects, between gray matter amyloid binding SUVR and ventricular tracer $\mathrm{AUC}_{35-80 \mathrm{~min}}, \rho=0.93, P<$ $0.01(\mathrm{~A})$, and rate of ventricular tracer clearance, $\rho=0.74, P<0.05$ (B). reason, ${ }^{11} \mathrm{C}-\mathrm{PiB}$, which shows binding in the nasal turbinates, was not used to estimate turbinate clearance. On the other hand, ${ }^{11} \mathrm{C}$-cocaine, which does not show cortical or turbinate binding, was valuable as a validator. In our study of ventricular CSF clearance, we controlled for the total tissue binding of THK5117 and concluded that the between-group clearance effect was largely unchanged. Similarly, we observed that THK5117 and ${ }^{11} \mathrm{C}-\mathrm{PiB}$ estimates of ventricular CSF clearance were highly correlated within subjects, a finding supportive of the method's precision when sampling the CSF directly.

Our estimates of nasal tracer concentrations are not precise because of the relatively poor spatial resolution and resultant partial-volume errors. Spatial resolution limitations are likely to have affected the tracer concentrations in the sparsely packed turbinate voxels, which did not show clearance reductions in AD. Spatial resolution also limited measurement of CSF clearance from the arachnoid granulations. On the other hand, the larger lateral ventricle provided robust CSF tracer concentration estimates and performed well as a clearance biomarker.

For the present, PET provides the only noninvasive strategy for estimating CSF clearance. Recently, Benveniste et al. reported using MRI that intrathecal contrast administration in an animal model could be used to image CSF bulk flow (10), but this approach is not feasible in clinical practice. Phase-contrast MRI is the only other noninvasive approach to estimate CSF flow in humans. However, it is increasingly recognized that the results are imprecise and of limited clinical utility because of slow CSF velocity rates (12). Overall, despite suboptimal tracers and obvious spatial resolution limitations, we provide the first evidence that dynamic PET yields important insight into CSF clearance. Our results highlight the importance of a ventricular clearance biomarker and suggest exploiting the nasal pathway for the sampling CSF molecules cleared from the brain. Finally, we view our study as a preliminary proof of concept, needing larger sample sizes, test-retest reliability, and longitudinal observations. Future studies will be served by developing comprehensive PET 
models that consider CSF flow in addition to estimating tracer uptake into tissue.

\section{CONCLUSION}

Dynamic PET was used to estimate in humans the CSF clearance in ventricular and extracranial regions. As in other mammals, we find that the human nasal turbinates are part of the CSF egress system. Ventricular CSF and superior nasal turbinate clearance measures were reduced in $\mathrm{AD}$ in comparison to control. Consistent with the clearance hypothesis of AD, ventricular CSF clearance was inversely related to $\mathrm{A} \beta$ deposition. PET-based CSF clearance measures may be of interest in the evaluation of the propagation and clearance of $A \beta$ and other abnormal proteins.

\section{DISCLOSURE}

This study was supported by NIH/NIA grants AG035137, AG032554, AG022374, AG13616, AG12101, AG08051, NIH-HLB HL111724, and HL118624 and Cohen Veterans Bioscience. The work at Tohoku University was supported by Health and Labor Sciences research grants from the Ministry of Health, Labor, and Welfare of Japan, a Grant-in-Aid for Scientific Research (B) (23390297), a Grant-inAid for Scientific Research on Innovative Areas (26117003), a grant from the Japan Advanced Molecular Imaging Program (J-AMP) of the Ministry of Education, Culture, Sports, Science and Technology, and the research fund from GE Healthcare and Sumitomo Electric Industries, Ltd. Mony J. de Leon, Yi Li, and Henry Rusinek applied for a patent to be assigned to NYU, based on this work. No other potential conflict of interest relevant to this article was reported.

\section{ACKNOWLEDGMENTS}

We offer a very special thanks to Professor Roy Weller, U. of Southampton, U.K., and Charles Nicholson, NYU Professor Emeritus, for their insightful comments and to Drs. Esther Fisher, Yeona Kang, and Edouardo Honig for their expert participation in the early stages of this project. We are most grateful to Drs. Nora D. Volkow and Gene-Jack Wang from the National Institute on Drug Abuse, $\mathrm{NIH}$, Bethesda, Maryland, for contributing the ${ }^{11} \mathrm{C}$-cocaine images.

\section{REFERENCES}

1. Hardy J, Selkoe DJ. The amyloid hypothesis of Alzheimer's disease: progress and problems on the road to therapeutics. Science. 2002;297:353-356.

2. Silverberg GD, Heit G, Huhn S, et al. The cerebrospinal fluid production rate is reduced in dementia of the Alzheimer's type. Neurology. 2001;57:1763-1766.

3. Ethell DW. Disruption of cerebrospinal fluid flow through the olfactory system may contribute to Alzheimer's disease pathogenesis. J Alzheimers Dis. 2014;41:1021-1030.

4. Tarasoff-Conway JM, Carare RO, Osorio RS, et al. Clearance systems in the brain: implications for Alzheimer disease. Nat Rev Neurol. 2015;11:457-470.

5. Kress BT, Iliff JJ, Xia M, et al. Impairment of paravascular clearance pathways in the aging brain. Ann Neurol. 2014;76:845-861.

6. Peng W, Achariyar TM, Li B, et al. Suppression of glymphatic fluid transport in a mouse model of Alzheimer's disease. Neurobiol Dis. 2016;93:215-225.

7. Kida S, Pantazis A, Weller RO. CSF drains directly from the subarachnoid space into nasal lymphatics in the rat: anatomy, histology and immunological significance. Neuropathol Appl Neurobiol. 1993;19:480-488.

8. Weller RO, Djuanda E, Yow HY, Carare RO. Lymphatic drainage of the brain and the pathophysiology of neurological disease. Acta Neuropathol (Berl). 2009;117:1-14.

9. Carare RO, Bernardes-Silva M, Newman TA, et al. Solutes, but not cells, drain from the brain parenchyma dextran: flouspheres, cerebral amyloid angiopathy and neuroimmunology. Neuropathol Appl Neurobiol. 2008;34:131-144.

10. Illiff JJ, Lee H, Yu M, et al. Brain-wide pathway for waste clearance captured by contrast-enhanced MRI. J Clin Invest. 2013;123:1299-1309.
11. Nagra G, Koh L, Zakharov A, Armstrong D, Johnston M. Quantification of cerebrospinal fluid transport across the cribriform plate into lymphatics in rats. Am J Physiol Regul Integr Comp Physiol. 2006;291:R1383-R1389.

12. Hladky SB, Barrand MA. Mechanisms of fluid movement into, through and out of the brain: evaluation of the evidence. Fluids Barriers CNS. 2014;11:26.

13. Johnston M, Zakharov A, Papaiconomou C, Salmasi G, Armstrong D. Evidence of connections between cerebrospinal fluid and nasal lymphatic vessels in humans, non-human primates and other mammalian species. Cerebrospinal Fluid Res. 2004;1:2.

14. Pan WR, Suami H, Corlett RJ, Ashton MW. Lymphatic drainage of the nasal fossae and nasopharynx: preliminary anatomical and radiological study with clinical implications. Head Neck. 2009;31:52-57.

15. McKhann GM, Knopman DS, Chertkow H, et al. The diagnosis of dementia due to Alzheimer's disease: recommendations from the National Institute on AgingAlzheimer's Association workgroups on diagnostic guidelines for Alzheimer's disease. Alzheimers Dement. 2011;7:263-269.

16. Okamura N, Furumoto S, Harada R, et al. Novel ${ }^{18} \mathrm{~F}$-labeled arylquinoline derivatives for noninvasive imaging of tau pathology in Alzheimer disease. $\mathrm{J} \mathrm{Nucl}$ Med. 2013;54:1420-1427.

17. Klunk WE, Engler H, Nordberg A, et al. Imaging brain amyloid in Alzheimer's disease with Pittsburgh compound-B. Ann Neurol. 2004;55:306-319.

18. Price JC, Klunk WE, Lopresti BJ, et al. Kinetic modeling of amyloid binding in humans using PET imaging and Pittsburgh compound-B. J Cereb Blood Flow Metab. 2005;25:1528-1547.

19. Matsumoto K, Kitamura K, Mizuta T, et al. Performance characteristics of a new 3-dimensional continuous-emission and spiral-transmission high-sensitivity and high-resolution PET camera evaluated with the NEMA NU 2-2001 standard. J Nucl Med. 2006;47:83-90.

20. Schwarz CG, Senjem ML, Gunter JL, et al. Optimizing PiB-PET SUVR changeover-time measurement by a large-scale analysis of longitudinal reliability, plausibility, separability, and correlation with MMSE. Neuroimage. 2017;144(part A):113-127.

21. Su Y, Blazey TM, Snyder AZ, et al. Quantitative amyloid imaging using imagederived arterial input function. PLoS One. 2015;10:e0122920.

22. Lee MH, Smyser CD, Shimony JS. Resting-state fMRI: a review of methods and clinical applications. AJNR. 2013;34:1866-1872.

23. Jonasson $\mathrm{M}$, Wall $\mathrm{A}$, Chiotis $\mathrm{K}$, et al. Tracer kinetic analysis of $(S){ }^{18} \mathrm{~F}-$ THK5117 as a PET tracer for assessing tau pathology. J Nucl Med. 2016; 57:574-581

24. Li Y, Tsui W, Rusinek H, et al. Cortical laminar binding of PET amyloid and tau tracers in Alzheimer disease. J Nucl Med. 2015;56:270-273.

25. Quade D. Rank analysis of covariance. J Am Stat Assoc. 1967;62:1187-1200.

26. Holm S. A simple sequentially rejective multiple test procedure. Scand J Stat. 1970;6:65-70.

27. Pollay M. The function and structure of the cerebrospinal fluid outflow system. Cerebrospinal Fluid Res. 2010;7:9-20.

28. Weller RO, Kida S, Zhang ET. Pathways of fluid drainage from the brain: morphological aspects and immunological significance in rat and man. Brain Pathol. 1992;2:277-284.

29. Lochhead JJ, Wolak DJ, Pizzo ME, Thorne RG. Rapid transport within cerebral perivascular spaces underlies widespread tracer distribution in the brain after intranasal administration. J Cereb Blood Flow Metab. 2015;35:371-381.

30. Bateman RJ, Munsell LY, Morris JC, Swarm R, Yarasheski KE, Holtzman DM. Human amyloid-beta synthesis and clearance rates as measured in cerebrospinal fluid in vivo. Nat Med. 2006;12:856-861.

31. Mawuenyega KG, Sigurdson W, Ovod V, et al. Decreased clearance of CNS beta-amyloid in Alzheimer's disease. Science. 2010;330:1774.

32. Kang JE, Lim MM, Bateman RJ, et al. Amyloid-beta dynamics are regulated by orexin and the sleep-wake cycle. Science. 2009;326:1005-1007.

33. Cirrito JR, Yamada KA, Finn MB, et al. Synaptic activity regulates interstitial fluid amyloid-beta levels in vivo. Neuron. 2005;48:913-922.

34. Minter MR, Taylor JM, Crack PJ. The contribution of neuroinflammation to amyloid toxicity in Alzheimer's disease. J Neurochem. 2016;136:457-474.

35. Iliff JJ, Wang M, Zeppenfeld DM, et al. Cerebral arterial pulsation drives paravascular CSF interstitial fluid exchange in the murine brain. J Neurosci. 2013;33:18190-18199.

36. Tolppanen AM, Solomon A, Kulmala J, et al. Leisure-time physical activity from mid- to late life, body mass index, and risk of dementia. Alzheimers Dement. 2015;11:434-443.e6.

37. Silverberg GD, Mayo M, Saul T, Fellmann J, Carvalho J, McGuire D. Continuous CSF drainage in AD: results of a double-blind, randomized, placebo-controlled study. Neurology. 2008;71:202-209.

38. Sepulcre J, Schultz AP, Sabuncu, Johnson KA. In vivo tau, amyloid, and gray matter profiles in the aging brain. $J$ Neurosci. 2016;36:7364-7374. 